\title{
Trigonometric Representations of Generalized Dedekind and Hardy Sums via the Discrete Fourier Transform*
}

\author{
Michael Th. Rassias and László Tóth \\ To Professor Helmut Maier on his 60th birthday
}

\begin{abstract}
We introduce some new higher dimensional generalizations of the Dedekind sums associated with the Bernoulli functions and of those Hardy sums which are defined by the sawtooth function. We generalize a variant of Parseval's formula for the discrete Fourier transform to derive finite trigonometric representations for these sums in a simple unified manner. We also consider a related sum involving the Hurwitz zeta function.
\end{abstract}

2010 Mathematics Subject Classification: 11F20, 11L03

Key Words and Phrases: Dedekind sums; Hardy sums; Bernoulli polynomials and functions; Hurwitz zeta function; discrete Fourier transform

\section{Introduction}

The classical Dedekind sum is defined for $h \in \mathbb{Z}, k \in \mathbb{N}:=\{1,2, \ldots\}$ by

$$
s(h, k):=\sum_{a(\bmod k)}\left(\left(\frac{a}{k}\right)\right)\left(\left(\frac{a h}{k}\right)\right),
$$

adopting the usual notation

$$
((x)):= \begin{cases}\{x\}-\frac{1}{2}, & \text { if } x \notin \mathbb{Z}, \\ 0, & \text { if } x \in \mathbb{Z},\end{cases}
$$

where $\{x\}:=x-\lfloor x\rfloor$ stands for the fractional part of $x$ (cf. [9], [13]). If $\operatorname{gcd}(h, k)=1$, then $s(h, k)$ can be represented as

$$
s(h, k)=\frac{1}{4 k} \sum_{a=1}^{k-1} \cot \left(\frac{\pi a}{k}\right) \cot \left(\frac{\pi a h}{k}\right)
$$

and

$$
s(h, k)=\frac{1}{2 \pi} \sum_{\substack{r=1 \\ r \neq 0(\bmod k)}}^{\infty} \frac{1}{r} \cot \left(\frac{\pi r h}{k}\right) .
$$

The identities (1) and (2) were derived in 1933 by H. Rademacher [12] in order to obtain a simple direct proof of the reciprocity formula for the Dedekind sums. See also [13, pp.

*In: Analytic Number Theory - In Honor of Helmut Maier's 60th Birthday, C. Pomerance and M. Th. Rassias (eds.), Springer, New York, 2015, pp. 329-343. 
18-25]. According to [1, p. 347], (1) was obtained earlier, in 1923 by H. Mellin. The identity (1) is also the starting point for various generalizations of $s(h, k)$. See, e.g., the papers of M. Beck [2], U. Dieter [8], D. Zagier [16].

It is known that (1) is a direct consequence of a variant of Parseval's formula for the discrete Fourier transform (DFT). See the paper of G. Almkvist [1, Sect. 6] and the book by M. Beck and S. Robins [4, Ch. 7]. More specifically, consider a function $f: \mathbb{Z} \rightarrow \mathbb{C}$, which is $k$-periodic (periodic with period $k$ ), where $k \in \mathbb{N}$. We define the DFT of $f$ as the function $\widehat{f}=\mathcal{F}(f)$, given by

$$
\widehat{f}(n):=\sum_{a(\bmod k)} f(a) e^{-2 \pi i a n / k} \quad(n \in \mathbb{Z}) .
$$

Furthermore, if $f_{1}$ and $f_{2}$ are $k$-periodic functions, then their inner product is

$$
\left\langle f_{1}, f_{2}\right\rangle:=\sum_{a(\bmod k)} f_{1}(a) \overline{f_{2}(a)},
$$

having the property

$$
\left\langle f_{1}, f_{2}\right\rangle=\frac{1}{k}\left\langle\widehat{f}_{1}, \widehat{f_{2}}\right\rangle
$$

or equivalently,

$$
\sum_{a(\bmod k)} f_{1}(a) f_{2}(-a)=\frac{1}{k} \sum_{a(\bmod k)} \widehat{f}_{1}(a) \widehat{f}_{2}(a) .
$$

Now, (1) follows by applying (3) to the functions

$$
f_{1}(a)=\left(\left(\frac{a}{k}\right)\right), f_{2}(a)=\left(\left(\frac{a h}{k}\right)\right)
$$

and using the fact that the DFT of the sawtooth function is essentially the cotangent function.

It is the aim of this paper to exploit this idea in order to deduce similar finite trigonometric representations for certain new generalized Dedekind and Hardy sums, in a simple unified manner. Our results are direct applications of a higher dimensional version of the identity (3), included in Theorem 2.1. We derive in this way Zagier-type identities for new higher dimensional generalizations of the Dedekind sums associated to the Bernoulli functions and of those Hardy sums which are defined by the sawtooth function. Note that all finite trigonometric representations we obtain contain only the tangent and cotangent functions, and are special cases of the Dedekind cotangent sums investigated by M. Beck [2]. Therefore the reciprocity law proved in $[2$, Th. 2] can be applied for each sum.

Furthermore, we consider a related sum, studied by M. Mikolás [11], involving the Hurwitz zeta function. We remark that (3) was used to evaluate some finite trigonometric and character sums, but not Dedekind and related sums, by M. Beck and M. Halloran [3]. We point out that the identity (2) can be obtained from (1) using another general result (Lemma 4.2 in Section 4).

\section{Properties of the DFT}

We will apply the following general result.

Theorem 2.1. Let $f_{1}, \ldots, f_{m}$ be arbitrary $k$-periodic functions and let $h_{j} \in \mathbb{Z}, \operatorname{gcd}\left(h_{j}, k\right)=1$ $(1 \leq j \leq m)$, where $m, k \in \mathbb{N}$. Then

$$
\sum_{\substack{a_{1}, \ldots, a_{m}(\bmod k) \\ a_{1}+\ldots+a_{m} \equiv 0(\bmod k)}} f_{1}\left(a_{1} h_{1}\right) \cdots f_{m}\left(a_{m} h_{m}\right)=\frac{1}{k} \sum_{a(\bmod k)} \widehat{f}_{1}\left(a h_{1}^{\prime}\right) \cdots \widehat{f_{m}}\left(a h_{m}^{\prime}\right),
$$

where $h_{j}^{\prime}$ is the multiplicative inverse of $h_{j}(\bmod k)$, that is $h_{j} h_{j}^{\prime} \equiv 1(\bmod k)$ with $1 \leq j \leq m$. 
Proof. We only need some simple well known facts concerning the DFT. See, for instance [15, Ch. 2], [4, Ch. 7]. The Cauchy convolution of the $k$-periodic functions $f_{1}$ and $f_{2}$ is defined by

$$
\left(f_{1} \otimes f_{2}\right)(n):=\sum_{\substack{a_{1}, a_{2}(\bmod k) \\ a_{1}+a_{2} \equiv n(\bmod k)}} f_{1}\left(a_{1}\right) f_{2}\left(a_{2}\right)=\sum_{a(\bmod k)} f_{1}(a) f_{2}(n-a) \quad(n \in \mathbb{Z}),
$$

which is associative and commutative. Also,

$$
\widehat{f_{1} \otimes f_{2}}=\widehat{f_{1}} \widehat{f}_{2} .
$$

More generally, if $f_{1}, \ldots, f_{m}$ are $k$-periodic functions, then

$$
\mathcal{F}\left(f_{1} \otimes \cdots \otimes f_{m}\right)=\mathcal{F}\left(f_{1}\right) \cdots \mathcal{F}\left(f_{m}\right) .
$$

Recalling that

$$
\mathcal{F}(\mathcal{F}(f))(n)=k f(-n) \quad(n \in \mathbb{Z}),
$$

which is valid for every $k$-periodic $f$, this yields

$$
k\left(f_{1} \otimes \cdots \otimes f_{m}\right)(-n)=\mathcal{F}\left(\mathcal{F}\left(f_{1}\right) \cdots \mathcal{F}\left(f_{m}\right)\right)(n),
$$

that is

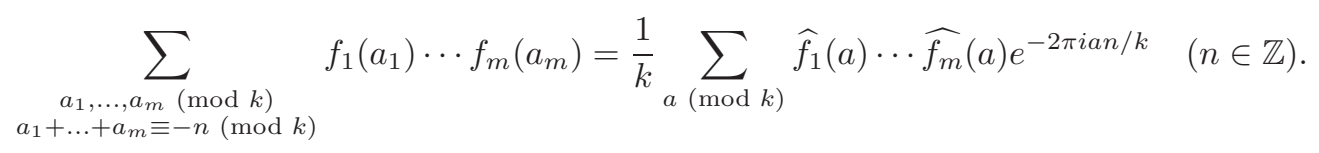

For $n=0$ we obtain

$$
\sum_{\substack{a_{1}, \ldots, a_{m}(\bmod k) \\ a_{1}+\ldots+a_{m} \equiv 0(\bmod k)}} f_{1}\left(a_{1}\right) \cdots f_{m}\left(a_{m}\right)=\frac{1}{k} \sum_{a(\bmod k)} \widehat{f_{1}}(a) \cdots \widehat{f_{m}}(a) .
$$

Now the result follows from (4) by showing the following property: Let $f$ be a $k$-periodic function and let $h \in \mathbb{Z}$ such that $\operatorname{gcd}(h, k)=1$. Then the DFT of the function $g$ defined by $g(n)=f(n h)(n \in \mathbb{Z})$ is $\widehat{g}(n)=\widehat{f}\left(n h^{\prime}\right)(n \in \mathbb{Z})$, where $h^{\prime}$ is the multiplicative inverse of $h$ $(\bmod k)$.

Indeed,

$$
\widehat{g}(n)=\sum_{a(\bmod k)} g(a) e^{-2 \pi i a n / k}=\sum_{a(\bmod k)} f(a h) e^{-2 \pi i a h h^{\prime} n / k},
$$

and since $\operatorname{gcd}(h, k)=1$, if $a$ runs through a complete system of residues $(\bmod k)$, then so does $b=a h$. Therefore,

$$
\widehat{g}(n)=\sum_{b(\bmod k)} f(b) e^{-2 \pi i b h^{\prime} n / k}=\widehat{f}\left(n h^{\prime}\right) .
$$

Corollary 2.1. Let $f_{1}$ and $f_{2}$ be $k$-periodic functions $(k \in \mathbb{N})$ and let $h_{1}, h_{2} \in \mathbb{Z}, \operatorname{gcd}\left(h_{1}, k\right)=$ $\operatorname{gcd}\left(h_{2}, k\right)=1$. Then

$$
\sum_{a(\bmod k)} f_{1}\left(a h_{1}\right) f_{2}\left(a h_{2}\right)=\frac{1}{k} \sum_{a(\bmod k)} \widehat{f}_{1}\left(-a h_{2}\right) \widehat{f}_{2}\left(a h_{1}\right) .
$$


Proof. Apply Theorem 2.1 for $m=2$. We deduce that

$$
\begin{aligned}
\sum_{a(\bmod k)} f_{1}\left(a h_{1}\right) f_{2}\left(-a h_{2}\right) & =\frac{1}{k} \sum_{a(\bmod k)} \widehat{f}_{1}\left(a h_{1}^{\prime}\right) \widehat{f}_{2}\left(a h_{2}^{\prime}\right) \\
& =\frac{1}{k} \sum_{a(\bmod k)} \widehat{f}_{1}\left(a h_{1}^{\prime} h_{2}^{\prime} h_{2}\right) \widehat{f}_{2}\left(a h_{1}^{\prime} h_{2}^{\prime} h_{1}\right) \\
& =\frac{1}{k} \sum_{b(\bmod k)} \widehat{f}_{1}\left(b h_{2}\right) \widehat{f}_{2}\left(b h_{1}\right)
\end{aligned}
$$

by using the fact that if $a$ runs through a complete system of residues $(\bmod k)$, then so does $b=a h_{1}^{\prime} h_{2}^{\prime}$, since $\operatorname{gcd}\left(h_{1} h_{2}, k\right)=1$. This gives (5) by setting $h_{2}:=-h_{2}$.

For $h_{1}=1, h_{2}=-1$ from (5) we derive (3).

Corollary 2.2. Let $f_{1}$ and $f_{2}$ be $k$-periodic functions $(k \in \mathbb{N})$ and assume that $f_{1}$ or $f_{2}$ is odd (resp. even). Let $h_{1}, h_{2} \in \mathbb{Z}, \operatorname{gcd}\left(h_{1}, k\right)=\operatorname{gcd}\left(h_{2}, k\right)=1$. Then

$$
\sum_{a(\bmod k)} f_{1}\left(a h_{1}\right) f_{2}\left(a h_{2}\right)=\frac{(-1)^{s}}{k} \sum_{a(\bmod k)} \widehat{f}_{1}\left(a h_{2}\right) \widehat{f}_{2}\left(a h_{1}\right),
$$

where $s=1$ if $f_{1}$ or $f_{2}$ is odd, $s=0$ if $f_{1}$ or $f_{2}$ is even.

Note that if the function $f$ is odd (resp. even), then $\widehat{f}$ is also odd (resp. even). If one of the functions $f_{1}, f_{2}$ is odd and the other one is even, then both sides of (6) are zero.

In this paper we will use the following DFT pairs of $k$-periodic functions.

Lemma 2.1. (i) Let $k \in \mathbb{N}$. The DFT of the $k$-periodic odd function $f(n)=\left(\left(\frac{n}{k}\right)\right)(n \in \mathbb{Z})$ is

$$
\widehat{f}(n)= \begin{cases}\frac{i}{2} \cot \left(\frac{\pi n}{k}\right), & \text { if } k \nmid n, \\ 0, & \text { if } k \mid n .\end{cases}
$$

(ii) Let $k \in \mathbb{N}$ and let $\overline{B_{r}}(r \in \mathbb{N})$ be the Bernoulli functions (cf. Section 3.1). The DFT of the k-periodic function $f(n)=\bar{B}_{r}\left(\frac{n}{k}\right)(n \in \mathbb{Z})$ is

$$
\widehat{f}(n)= \begin{cases}r k^{1-r}\left(\frac{i}{2}\right)^{r} \cot ^{(r-1)}\left(\frac{\pi n}{k}\right), & \text { if } k \nmid n, \\ B_{r} k^{1-r}, & \text { if } k \mid n,\end{cases}
$$

where $B_{r}$ is the $r$-th Bernoulli number and $\cot ^{(m)}$ is the $m$-th derivative of the cotangent function.

(iii) Let $k \in \mathbb{N}$ be even. The DFT of the $k$-periodic odd function $f(n)=(-1)^{n}\left(\left(\frac{n}{k}\right)\right)$ $(n \in \mathbb{Z})$ is

$$
\widehat{f}(n)= \begin{cases}-\frac{i}{2} \tan \left(\frac{\pi n}{k}\right), & \text { if } n \not \equiv \frac{k}{2}(\bmod k), \\ 0, & \text { if } n \equiv \frac{k}{2}(\bmod k) .\end{cases}
$$

(iv) Let $k$ be odd and let $n(\bmod k)=n-k\lfloor n / k\rfloor$ be the least nonnegative residue of $n$ $(\bmod k)$. The DFT of the $k$-periodic odd function

$$
f(n)= \begin{cases}(-1)^{n(\bmod k)}, & \text { if } k \nmid n, \\ 0, & \text { if } k \mid n\end{cases}
$$

is

$$
\widehat{f}(n)=i \tan \left(\frac{\pi n}{k}\right) \quad(n \in \mathbb{Z}) .
$$


(v) Let $k \in \mathbb{N}$. Let $F(s, x), \zeta(s, x)$ and $\zeta(s)$ be the periodic zeta function, the Hurwitz zeta function, and the Riemann zeta function, respectively (cf. Section 3.3). For $\Re s>1$ the DFT of the $k$ periodic function $f(n)=F\left(s, \frac{n}{k}\right)(n \in \mathbb{Z})$ is

$$
\widehat{f}(n)= \begin{cases}k^{1-s} \zeta\left(s,\left\{\frac{n}{k}\right\}\right), & \text { if } k \nmid n, \\ k^{1-s} \zeta(s), & \text { if } k \mid n .\end{cases}
$$

Here (i) and (iv) are well known. They follow, together with (iii) and (v), by easy computations from the definition of the DFT. For (ii) we refer to [2, Lemma 6]. See also [1, Sect. 6].

\section{Applications}

\subsection{Generalized Dedekind sums}

We first derive the following higher dimensional generalization of the identity (1), first deduced by D. Zagier [16, Th. p. 157], in a slightly different form, by applying some other arguments.

Theorem 3.2. Let $k \in \mathbb{N}, m \in \mathbb{N}$ be even and let $h_{j} \in \mathbb{Z}, \operatorname{gcd}\left(h_{j}, k\right)=1(1 \leq j \leq m)$. Then

$$
\begin{aligned}
& \sum_{\substack{a_{1}, \ldots, a_{m}(\bmod k) \\
a_{1}+\ldots+a_{m} \equiv 0(\bmod k)}}\left(\left(\frac{a_{1} h_{1}}{k}\right)\right) \cdots\left(\left(\frac{a_{m} h_{m}}{k}\right)\right) \\
& =\frac{(-1)^{m / 2}}{2^{m} k} \sum_{a=1}^{k-1} \cot \left(\frac{\pi a h_{1}^{\prime}}{k}\right) \cdots \cot \left(\frac{\pi a h_{m}^{\prime}}{k}\right) .
\end{aligned}
$$

Proof. Apply Theorem 2.1 for $f_{1}=\ldots=f_{m}=f$, where $f(n)=\left(\left(\frac{n}{k}\right)\right)(n \in \mathbb{Z})$. Use Lemma $2.1 /(\mathrm{i})$.

Note that if $m$ is odd, then both sides of (7) are zero.

Corollary 3.3. Assume that $m=2$. Let $k \in \mathbb{N}, h_{1}, h_{2} \in \mathbb{Z}, \operatorname{gcd}\left(h_{1}, k\right)=\operatorname{gcd}\left(h_{2}, k\right)=1$. Then

$$
\sum_{a=1}^{k-1}\left(\left(\frac{a h_{1}}{k}\right)\right)\left(\left(\frac{a h_{2}}{k}\right)\right)=\frac{1}{4 k} \sum_{a=1}^{k-1} \cot \left(\frac{\pi a h_{1}}{k}\right) \cot \left(\frac{\pi a h_{2}}{k}\right) .
$$

Identity (8) is the homogeneous version of (1) and is equivalent to (1).

Now consider the Bernoulli polynomials $B_{r}(x)(r \geq 0)$, defined by

$$
\frac{t e^{x t}}{e^{t}-1}=\sum_{r=0}^{\infty} \frac{B_{r}(x)}{r !} t^{r}
$$

Here

$$
B_{1}(x)=x-1 / 2, B_{2}(x)=x^{2}-x+1 / 6, B_{3}(x)=x^{3}-3 x^{2} / 2+x / 2 \text { and } B_{r}:=B_{r}(0)
$$

are the Bernoulli numbers. The Bernoulli functions $x \mapsto \bar{B}_{r}(x)$ are given by

$$
\bar{B}_{r}(x)=B_{r}(\{x\}) \quad(x \in \mathbb{R}) .
$$

Note that

$$
\bar{B}_{1}(x)=((x)) \text { for } x \notin \mathbb{Z}
$$


but

$$
\bar{B}_{1}(x)=-1 / 2 \neq 0=((x)) \text { for } x \in \mathbb{Z} .
$$

For $r_{1}, \ldots, r_{m} \in \mathbb{N}, h_{1}, \ldots, h_{m} \in \mathbb{Z}$ we define the higher dimensional Dedekind-Bernoulli sum by

$$
s_{r_{1}, \ldots, r_{m}}\left(h_{1}, \ldots, h_{m} ; k\right):=\sum_{\begin{array}{c}
a_{1}, \ldots, a_{m}(\bmod k) \\
a_{1}+\ldots+a_{m} \equiv 0(\bmod k)
\end{array}} \bar{B}_{r_{1}}\left(\frac{a_{1} h_{1}}{k}\right) \cdots \bar{B}_{r_{m}}\left(\frac{a_{m} h_{m}}{k}\right) .
$$

In the case when $m=2$ and by setting $h_{2}:=-h_{2}$ we obtain the sum

$$
s_{r_{1}, r_{2}}\left(h_{1},-h_{2} ; k\right):=\sum_{a(\bmod k)} \bar{B}_{r_{1}}\left(\frac{a h_{1}}{k}\right) \bar{B}_{r_{2}}\left(\frac{a h_{2}}{k}\right),
$$

first investigated by L. Carlitz [7] and M. Mikolás [11]. See the paper of M. Beck [2] for further historical remarks.

Theorem 3.3. Let $k, m, r_{j} \in \mathbb{N}$ be such that $A:=r_{1}+\ldots+r_{m}$ is even and $h_{j} \in \mathbb{Z}$, $\operatorname{gcd}\left(h_{j}, k\right)=1(1 \leq j \leq m)$. Then

$$
\begin{gathered}
s_{r_{1}, \ldots, r_{m}}\left(h_{1}, \ldots, h_{m} ; k\right)=\frac{B_{r_{1}} \cdots B_{r_{m}}}{k^{A-m+1}} \\
+\frac{(-1)^{A / 2} r_{1} \cdots r_{m}}{2^{A} k^{A-m+1}} \sum_{a=1}^{k-1} \cot ^{\left(r_{1}-1\right)}\left(\frac{\pi a h_{1}^{\prime}}{k}\right) \cdots \cot ^{\left(r_{m}-1\right)}\left(\frac{\pi a h_{m}^{\prime}}{k}\right),
\end{gathered}
$$

Note that if $A$ is odd, then the sum in (11) vanishes. If $A$ is odd and there is at least one $j$ such that $r_{j} \geq 3$, then $B_{r_{j}}=0$ and the sum (9) vanishes as well.

Proof. Apply Theorem 2.1 and Lemma 2.1/(ii) to the functions

$$
f_{j}(n)=\bar{B}_{r_{j}}\left(\frac{n}{k}\right) \quad(1 \leq j \leq m) .
$$

Corollary 3.4. ([2, Cor. 7]) Let $k, r_{1}, r_{2} \in \mathbb{N}, h_{1}, h_{2} \in \mathbb{Z}$ be such that $r_{1}+r_{2}$ is even and $\operatorname{gcd}\left(h_{1}, k\right)=\operatorname{gcd}\left(h_{2}, k\right)=1$. Then

$$
\begin{gathered}
\sum_{a(\bmod k)} \bar{B}_{r_{1}}\left(\frac{a h_{1}}{k}\right) \bar{B}_{r_{2}}\left(\frac{a h_{2}}{k}\right) \\
=\frac{B_{r_{1}} B_{r_{2}}}{k^{r_{1}+r_{2}-1}}+\frac{(-1)^{\left(r_{1}-r_{2}\right) / 2} r_{1} r_{2}}{2^{r_{1}+r_{2}} k^{r_{1}+r_{2}-1}} \sum_{a=1}^{k-1} \cot ^{\left(r_{1}-1\right)}\left(\frac{\pi a h_{1}}{k}\right) \cot ^{\left(r_{2}-1\right)}\left(\frac{\pi a h_{2}}{k}\right) .
\end{gathered}
$$




\subsection{Generalized Hardy sums}

The Hardy sums (known also as Hardy-Berndt sums) are defined for $h \in \mathbb{Z}, k \in \mathbb{N}$ as follows.

$$
\begin{aligned}
& S(h, k):=\sum_{a(\bmod k)}(-1)^{a+1+\lfloor a h / k\rfloor}, \\
& s_{1}(h, k):=\sum_{a(\bmod k)}(-1)^{\lfloor a h / k\rfloor}\left(\left(\frac{a}{k}\right)\right), \\
& s_{2}(h, k):=\sum_{a(\bmod k)}(-1)^{a}\left(\left(\frac{a}{k}\right)\right)\left(\left(\frac{a h}{k}\right)\right), \\
& s_{3}(h, k):=\sum_{a(\bmod k)}(-1)^{a}\left(\left(\frac{a h}{k}\right)\right), \\
& s_{4}(h, k):=\sum_{a(\bmod k)}(-1)^{\lfloor a h / k\rfloor}, \\
& s_{5}(h, k):=\sum_{a(\bmod k)}(-1)^{a+\lfloor a h / k\rfloor}\left(\left(\frac{a}{k}\right)\right) .
\end{aligned}
$$

B. C. Berndt and L. A. Goldberg [6] derived finite and infinite series representations for the above sums. These identities were also obtained later by R. Sitaramachandrarao [14] by using some different arguments. One could see $[2,6,8,14]$ for the history of these sums as well as for further results on the Hardy sums, including reciprocity formulas.

We define the following generalization of $s_{2}(h, k)$ :

$$
A\left(h_{1}, \ldots, h_{m} ; k\right):=\sum_{\substack{a_{1}, \ldots, a_{m}(\bmod k) \\ a_{1}+\ldots+a_{m} \equiv 0(\bmod k)}}(-1)^{a_{1}}\left(\left(\frac{a_{1} h_{1}}{k}\right)\right) \cdots\left(\left(\frac{a_{m} h_{m}}{k}\right)\right) .
$$

Theorem 3.4. Let $k, m \in \mathbb{N}$ be even, $h_{1}, \ldots, h_{m} \in \mathbb{Z}, h_{1}$ odd, $\operatorname{gcd}\left(h_{j}, k\right)=1(1 \leq j \leq m)$. Then

$$
A\left(h_{1}, \ldots, h_{m} ; k\right)=\frac{(-1)^{m / 2-1}}{2^{m} k} \sum_{\substack{1 \leq a \leq k-1 \\ a \neq k / 2}} \tan \left(\frac{\pi a h_{1}^{\prime}}{k}\right) \cot \left(\frac{\pi a h_{2}^{\prime}}{k}\right) \cdots \cot \left(\frac{\pi a h_{m}^{\prime}}{k}\right) .
$$

Proof. Let $f_{1}(n)=(-1)^{n}\left(\left(\frac{n}{k}\right)\right)$ and $f_{j}(n)=\left(\left(\frac{n}{k}\right)\right)(2 \leq j \leq m)$. Apply Theorem 2.1 and Lemma $2.1 /(\mathrm{i}),(\mathrm{iv})$.

Corollary 3.5. Assume that $m=2$. Let $k \in \mathbb{N}$ be even, $h_{1}, h_{2} \in \mathbb{Z}, h_{1}$ odd, and $\operatorname{gcd}\left(h_{1}, k\right)=$ $\operatorname{gcd}\left(h_{2}, k\right)=1$. Then

$$
\sum_{a=1}^{k-1}(-1)^{a}\left(\left(\frac{a h_{1}}{k}\right)\right)\left(\left(\frac{a h_{2}}{k}\right)\right)=-\frac{1}{4 k} \sum_{\substack{1 \leq a \leq k-1 \\ a \neq k / 2}} \tan \left(\frac{\pi a h_{2}}{k}\right) \cot \left(\frac{\pi a h_{1}}{k}\right) .
$$

In the case $m=2, h_{1}=1, h_{2}=h$ we obtain the following corollary, cf. [6, Eq. (14)], [14, Eq. (7.3)].

Corollary 3.6. If $k \in \mathbb{N}$ is even, $h \in \mathbb{Z}, \operatorname{gcd}(h, k)=1$, then

$$
s_{2}(h, k)=-\frac{1}{4 k} \sum_{\substack{1 \leq a \leq k-1 \\ a \neq k / 2}} \tan \left(\frac{\pi a h}{k}\right) \cot \left(\frac{\pi a}{k}\right) .
$$


Next, we define the following common generalization of $s_{1}(h, k), s_{3}(h, k)$ and $s_{5}(h, k)$, as follows.

$$
B\left(h_{1}, \ldots, h_{m} ; k\right):=\sum_{\substack{a_{1}, \ldots, a_{m}(\bmod k) \\ a_{1} \neq 0(\bmod k) \\ a_{1}+\ldots+a_{m} \equiv 0(\bmod k)}}(-1)^{a_{1} h_{1}+k\left\lfloor a_{1} h_{1} / k\right\rfloor}\left(\left(\frac{a_{2} h_{2}}{k}\right)\right) \cdots\left(\left(\frac{a_{m} h_{m}}{k}\right)\right) .
$$

Theorem 3.5. Let $k \in \mathbb{N}$ be odd, $m \in \mathbb{N}$ be even, $h_{j} \in \mathbb{Z}, \operatorname{gcd}\left(h_{j}, k\right)=1(1 \leq j \leq m)$. Then

$$
B\left(h_{1}, \ldots, h_{m} ; k\right)=\frac{(-1)^{m / 2}}{2^{m-1} k} \sum_{a=1}^{k-1} \tan \left(\frac{\pi a h_{1}^{\prime}}{k}\right) \cot \left(\frac{\pi a h_{2}^{\prime}}{k}\right) \cdots \cot \left(\frac{\pi a h_{m}^{\prime}}{k}\right) .
$$

Proof. Apply Theorem 2.1 to the following functions:

$$
\begin{aligned}
& f_{1}(n)= \begin{cases}(-1)^{n(\bmod k)}, & \text { if } k \nmid n, \\
0, & \text { if } k \mid n,\end{cases} \\
& f_{j}(n)=\left(\left(\frac{n}{k}\right)\right) \quad(2 \leq j \leq m)
\end{aligned}
$$

and also Lemma 2.1/(iv).

Corollary 3.7. Assume that $m=2$. Let $k \in \mathbb{N}$ be odd, $h_{1}, h_{2} \in \mathbb{Z}, h_{1}$ odd, $\operatorname{gcd}\left(h_{1}, k\right)=$ $\operatorname{gcd}\left(h_{2}, k\right)=1$. Then

$$
\sum_{a=1}^{k-1}(-1)^{a+\left\lfloor a h_{1} / k\right\rfloor}\left(\left(\frac{a h_{2}}{k}\right)\right)=\frac{1}{2 k} \sum_{a=1}^{k-1} \tan \left(\frac{\pi a h_{2}}{k}\right) \cot \left(\frac{\pi a h_{1}}{k}\right) .
$$

For $m=2$ in the special cases $h_{1}=1, h_{2}=h$ and $h_{1}=h, h_{2}=1$, respectively, we obtain the identities, cf. [6, Eq. (15), (17)], [14, Eq. (7.4), (7.6)], as follows.

Corollary 3.8. If $k \in \mathbb{N}$ is odd, $h \in \mathbb{Z}, \operatorname{gcd}(h, k)=1$, then

$$
s_{3}(h, k)=\frac{1}{2 k} \sum_{a=1}^{k-1} \tan \left(\frac{\pi a h}{k}\right) \cot \left(\frac{\pi a}{k}\right) .
$$

If $k \in \mathbb{N}$ is odd, $h \in \mathbb{Z}$ is odd, $\operatorname{gcd}(h, k)=1$, then

$$
s_{5}(h, k)=\frac{1}{2 k} \sum_{a=1}^{k-1} \tan \left(\frac{\pi a}{k}\right) \cot \left(\frac{\pi a h}{k}\right) .
$$

Corollary 3.9. Assume that $m=2$. Let $k \in \mathbb{N}$ be odd, $h_{1}, h_{2} \in \mathbb{Z}, h_{1}$ even, $\operatorname{gcd}\left(h_{1}, k\right)=$ $\operatorname{gcd}\left(h_{2}, k\right)=1$. Then

$$
\sum_{a=1}^{k-1}(-1)^{\left\lfloor a h_{1} / k\right\rfloor}\left(\left(\frac{a h_{2}}{k}\right)\right)=\frac{1}{2 k} \sum_{a=1}^{k-1} \tan \left(\frac{\pi a h_{2}}{k}\right) \cot \left(\frac{\pi a h_{1}}{k}\right) .
$$

For $m=2$ in the special case $h_{1}=h$ even, $h_{2}=1$ we obtain the identity, cf. [6, Eq. (13)], [14, Eq. (7.2)], as follows.

Corollary 3.10. If $k \in \mathbb{N}$ is odd, $h \in \mathbb{Z}$ is even, $\operatorname{gcd}(h, k)=1$, then

$$
s_{1}(h, k)=\frac{1}{2 k} \sum_{a=1}^{k-1} \tan \left(\frac{\pi a}{k}\right) \cot \left(\frac{\pi a h}{k}\right) .
$$


Note that the Hardy sums $S(h, k)$ and $s_{4}(h, k)$ can also be treated with the DFT in the case when $k$ is odd. For example, applying Corollary 2.2 to the functions

$$
f_{1}(n)=f_{2}(n)= \begin{cases}(-1)^{n(\bmod k)}, & \text { if } k \nmid n, \\ 0, & \text { if } k \mid n\end{cases}
$$

we obtain the following representation.

Corollary 3.11. If $k \in \mathbb{N}$ is odd, $h_{1}, h_{2} \in \mathbb{Z}, \operatorname{gcd}\left(h_{1}, k\right)=\operatorname{gcd}\left(h_{2}, k\right)=1$, then

$$
\sum_{a=1}^{k-1}(-1)^{a\left(h_{1}+h_{2}\right)(\bmod k)}=\frac{1}{k} \sum_{a=1}^{k-1} \tan \left(\frac{\pi a h_{1}}{k}\right) \tan \left(\frac{\pi a h_{2}}{k}\right) .
$$

If $h_{2}=1$ and $h_{1}=h$ is odd, then the left hand side of (14) is exactly $s_{4}(h, k)$. See $[6$, Eq. (16)], [14, Eq. (7.5)]. If $h_{1}=h_{2}=1$, then (14) provides the following classical identity, valid for $k \in \mathbb{N}$ odd, cf. [3, Prop. 3.1]:

$$
\sum_{a=1}^{k-1} \tan ^{2}\left(\frac{\pi a}{k}\right)=k^{2}-k
$$

Remark 3.1. For $k$ odd, $h$ even, the formula $[6$, Eq. (13)] receives the following representation:

$$
s_{1}(h, k)=-\frac{1}{2 k} \sum_{\substack{j=1 \\ j \neq(k+1) / 2}}^{k} \cot \left(\frac{\pi h(2 j-1)}{2 k}\right) \cot \left(\frac{\pi(2 j-1)}{2 k}\right),
$$

which can easily be transformed into

$$
s_{1}(h, k)=\frac{1}{k} \sum_{j=1}^{(k-1) / 2} \tan \left(\frac{\pi j}{k}\right) \cot \left(\frac{\pi h j}{k}\right)
$$

that is equal to the right hand side of (13). Similar considerations are valid for the corresponding formulas on the Hardy sums $s_{4}(h, k), s_{5}(h, k)$ and $S(h, k)$.

Remark 3.2. The finite sum identities (7.1), (7.2), (7.3), (7.5), and (7.6) from the paper [14] contain some misprints. Namely, in formulas (7.1) and (7.5) the sum $\sum_{r=1}^{k-1}$ should be $\sum_{r=1}^{k}$, while in (7.2) and (7.6) the sum $\sum_{r=1, r \neq(k+1) / 2}^{k-1}$ should be $\sum_{r=1, r \neq(k+1) / 2}^{k}$, the missing terms being nonzero. Furthermore, in formula (7.3) the sum $\sum_{r=1}^{k-1}$ should be $\sum_{r=1, r \neq k / 2}^{k-1}$, the term for $r=k / 2$ (namely $\tan (\pi / 2)$ ) being not defined.

One could possibly investigate some further higher dimensional generalizations and analogues of the Hardy sums involving the Bernoulli functions, however we do not discuss this in the present paper.

\subsection{Sums involving the Hurwitz zeta function}

Theorem 2.1 and its corollaries can be applied in several other situations as well. For example, let

$$
\zeta(s, x):=\sum_{n=0}^{\infty} \frac{1}{(n+x)^{s}}
$$

be the Hurwitz zeta function, where $0<x \leq 1$ and $\zeta(s, 1)=\zeta(s)$ is the Riemann zeta function. The function

$$
D(h, k):=\sum_{a=1}^{k-1} \zeta\left(s_{1},\left\{\frac{a h_{1}}{k}\right\}\right) \zeta\left(s_{2},\left\{\frac{a h_{2}}{k}\right\}\right)
$$


investigated by M. Mikolás [11], is an analogue of the Dedekind sum (10), taking into account that

$$
B_{n}(x)=-n \zeta(1-n, x) \quad(n \in \mathbb{N}, 0<x \leq 1) .
$$

Let

$$
F(s, x):=\sum_{n=1}^{\infty} \frac{e^{2 \pi i n x}}{n^{s}} \quad(x \in \mathbb{R})
$$

be the periodic zeta function, which converges for $\Re s>0$ if $x \notin \mathbb{Z}$ and for $\Re s>1$ if $x \in \mathbb{Z}$.

Applying Corollary 2.1 to the functions

$$
f_{j}(n)=F\left(s_{j}, \frac{n}{k}\right) \quad(n \in \mathbb{Z}, 1 \leq j \leq 2),
$$

we deduce by Lemma $2.1 /(\mathrm{v})$ the next new result.

Theorem 3.6. Let $k \in \mathbb{N}, h_{1}, h_{2} \in \mathbb{Z}, \operatorname{gcd}\left(h_{1}, k\right)=\operatorname{gcd}\left(h_{2}, k\right)=1$ and let $s_{1}, s_{2} \in \mathbb{C}$, $\Re s_{1}, \Re s_{2}>1$. Then

$$
D(h, k)=\left(k^{s_{1}+s_{2}-1}-1\right) \zeta\left(s_{1}\right) \zeta\left(s_{2}\right)+k^{s_{1}+s_{2}-1} \sum_{a=1}^{k-1} F\left(s_{1}, \frac{a h_{2}}{k}\right) F\left(s_{2},-\frac{a h_{1}}{k}\right) .
$$

\section{Some further remarks}

The following simple and useful result can be applied to obtain infinite series representations for the Dedekind and Hardy sums.

Lemma 4.2. If $f: \mathbb{N} \rightarrow \mathbb{C}$ is a $k$-periodic $(k \in \mathbb{N})$ odd function, then

$$
\begin{aligned}
S(f):=\sum_{r=1}^{\infty} \frac{f(r)}{r} & =\frac{\pi}{2 k} \sum_{r=1}^{k-1} f(r) \cot \left(\frac{\pi r}{k}\right) \\
& =-\frac{\pi i}{k^{2}} \sum_{r=1}^{k-1} r \widehat{f}(r) .
\end{aligned}
$$

For the Dedekind sum, (2) is a direct consequence of identities (16) and (1). As another example, (16) and (12) imply that for $k$ odd, $\operatorname{gcd}(h, k)=1$, one has

$$
s_{3}(h, k)=\frac{1}{\pi} \sum_{r=1}^{\infty} \frac{1}{r} \tan \left(\frac{\pi r h}{k}\right) .
$$

One could see [6, Th. 1], [14, Th. 7.1] for the above formula as well as for similar representations regarding Hardy sums.

Identity (16) of Lemma 4.2 was proved in [14, Lemma 2.1] by applying results of D. H. Lehmer [10] on the generalized Euler constants $\gamma(r, k)$ associated with the infinite arithmetic progression $r, r+k, r+2 k, \ldots(1 \leq r \leq k)$, where

$$
\gamma(r, k):=\lim _{x \rightarrow \infty}\left(\sum_{\substack{1 \leq n \leq x \\ n \equiv r(\bmod k)}} \frac{1}{n}-\frac{1}{k} \log x\right) .
$$

B. C. Berndt [5] deduced (17) by contour integration (with a different definition of the DFT). The fact that the finite sums (16) and (17) are equal, provides another simple consequence of Corollary 2.2, applied to the odd functions $f: \mathbb{N} \rightarrow \mathbb{C}$ and $n \mapsto\left(\left(\frac{n}{k}\right)\right)$. 
Furthermore, according to [10, Th. 8], if $f$ is a $k$-periodic function, then

$$
S(f)=\sum_{r=1}^{k} f(r) \gamma(r, k),
$$

provided that $\sum_{r=1}^{k} f(k)=0$, representing a necessary and sufficient condition for the convergence of the series $S(f)$ (which holds if $f$ is a $k$-periodic and odd function).

We note that the DFT of the $k$-periodic function $r \mapsto \gamma(r, k)$ is

$$
\widehat{\gamma}(r, k)= \begin{cases}F\left(1,-\frac{r}{k}\right), & \text { if } k \nmid r, \\ \gamma, & \text { if } k \mid r\end{cases}
$$

(cf. [10, p. 127]), where $F(s, x)$ is the periodic zeta function defined by $(15)$ and $\gamma:=\gamma(0,1)$ is Euler's constant. Therefore, we deduce by Corollary 2.1 the next identity.

Corollary 4.12. If $f: \mathbb{N} \rightarrow \mathbb{C}$ is a $k$-periodic $(k \in \mathbb{N})$ odd function, then

$$
S(f)=-\frac{1}{k} \sum_{r=1}^{k-1} \widehat{f}(r) F\left(1,-\frac{r}{k}\right) .
$$

Acknowledgement. The authors would like to thank the referee for useful remarks which helped improve the presentation of the paper.

\section{References}

[1] G. Almkvist, Asymptotic formulas and generalized Dedekind sums, Experiment. Math. 7 (1998), 343-359.

[2] M. Beck, Dedekind cotangent sums, Acta Arith. 109 (2003), 109-130.

[3] M. Beck and M. Halloran, Finite trigonometric character sums via discrete Fourier analysis, Int. J. Number Theory 6 (2010), 51-67.

[4] M. Beck and S. Robins, Computing the Continuous Discretely: Integer-Point Enumeration in Polyhedra, Undergrad. Texts Math., Springer, New York, 2007.

[5] B. C. Berndt, Solution to Problem E2719, Amer. Math. Monthly 86 (1979), 786-788.

[6] B. C. Berndt and L. A. Goldberg, Analytic properties of arithmetic sums arising in the theory of the classical theta-functions, SIAM J. Math. Anal. 15 (1984), 143-150.

[7] L. Carlitz, Some theorems on generalized Dedekind sums, Pacific J. Math. 3 (1953), $513-522$.

[8] U. Dieter, Cotangent sums, a further generalization of Dedekind sums, J. Number Theory 18 (1984), 289-305.

[9] H. Iwaniec and E. Kowalski, Analytic Number Theory, Amer. Math. Soc. Colloq., Providence, RI, Vol. 53, 2004.

[10] D. H. Lehmer, Euler constants for arithmetical progressions, Acta Arith. 27 (1975), 125-142.

[11] M. Mikolás, On certain sums generating the Dedekind sums and their reciprocity laws, Pacific J. Math. 7 (1957), 1167-1178.

[12] H. Rademacher, Egy reciprocitásképletről a modulfüggvények elméletéből, Mat. Fiz. Lapok 40 (1933), 24-34; Collected Papers II. pp. 26-37, MIT Press. Cambridge, Mass., 1974. 
[13] H. Rademacher and E. Grosswald, Dedekind Sums, in: Carus Math. Monograph, Vol. 16, Math. Assoc. America, Washington, DC, 1972.

[14] R. Sitaramachandrarao, Dedekind and Hardy sums, Acta Arith. 48 (1987), 325-340.

[15] A. Terras, Fourier Analysis on Finite Groups and Applications, London Mathematical Society Student Texts, Vol. 43, Cambridge University Press, Cambridge, 1999.

[16] D. Zagier, Higher dimensional Dedekind sums, Math. Ann. 202 (1973), 149-172.

Michael Th. Rassias

Department of Mathematics, ETH-Zürich, Rämistrasse 101, 8092 Zürich, Switzerland

Department of Mathematics, Princeton University, Fine Hall, Washington Road, Princeton, NJ 08544-1000, USA

E-mail: michail.rassias@math.ethz.ch, michailrassias@math.princeton.edu

László Tóth

Department of Mathematics, University of Pécs, Ifjúság útja 6, 7624 Pécs, Hungary

E-mail: ltoth@gamma.ttk.pte.hu 